\title{
Development of an efficient RNA interference method by feeding for the microcrustacean Daphnia
}

\author{
Charles A. Schumpert, Jeffry L. Dudycha and Rekha C. Patel ${ }^{*}$
}

\begin{abstract}
Background: RNA interference (RNAi) is an important molecular tool for analysis of gene function in vivo. Daphnia, a freshwater microcrustacean, is an emerging model organism for studying cellular and molecular processes involved in aging, development, and ecotoxicology especially in the context of environmental variation. However, in spite of the availability of a fully sequenced genome of Daphnia pulex, meaningful mechanistic studies have been hampered by a lack of molecular techniques to alter gene expression. A microinjection method for gene knockdown by RNAi has been described but the need for highly specialized equipment as well as technical expertise limits the wider application of this technique. In addition to being expensive and technically challenging, microinjections can only target genes expressed during embryonic stages, thus making it difficult to achieve effective RNAi in adult organisms.

Results: In our present study we present a bacterial feeding method for RNAi in Daphnia. We used a melanic Daphnia species (Daphnia melanica) that exhibits dark pigmentation to target phenoloxidase, a key enzyme in the biosynthesis of melanin. We demonstrate that our RNAi method results in a striking phenotype and that the phenoloxidase mRNA expression and melanin content, as well as survival following UV insults, are diminished as a result of RNAi.

Conclusions: Overall, our results establish a new method for RNAi in Daphnia that significantly advances further use of Daphnia as a model organism for functional genomics studies. The method we describe is relatively simple and widely applicable for knockdown of a variety of genes in adult organisms.
\end{abstract}

Keywords: RNA interference, Daphnia, Knockdown, Feeding, Melanin, Phenoloxidase

\section{Background}

In order to study gene function in intact organisms, effective techniques to manipulate gene expression to achieve an over-expression or knockdown are essential. RNA interference (RNAi) has revolutionized several fields of biology by making it possible to study loss-offunction effects in various organisms without the timeconsuming, laborious genetic manipulations. RNAi is a mechanism in which dsRNA molecules trigger gene silencing in a sequence-specific manner, usually resulting in degradation of the transcript complementary to one strand of the dsRNA ([1] and the references within). The

\footnotetext{
* Correspondence: patelr@biol.sc.edu

Department of Biological Sciences, University of South Carolina, 700 Sumter Street, Columbia, SC 29208, USA
}

(c) 2015 Schumpert et al. Open Access This article is distributed under the terms of the Creative Commons Attribution 4.0 International License (http://creativecommons.org/licenses/by/4.0/), which permits unrestricted use, distribution, and reproduction in any medium, provided you give appropriate credit to the original author(s) and the source, provide a link to the Creative Commons license, and indicate if changes were made. The Creative Commons Public Domain Dedication waiver (http://creativecommons.org/publicdomain/zero/1.0/) applies to the data made available in this article, unless otherwise stated.

RNAi pathway is conserved throughout eukaryotes with examples of the RNAi mechanism being used to silence gene expression in numerous model organisms including (but not limiting to) Schizosaccharomyces pombe, Tetrahymena, Drosophila melanogaster, Caenorhabditis elegans, Danio rerio, Xenopus, and Mus musculus [2]. This conserved mechanism of gene silencing has led to exceptional use of reverse genetics methods and has led to a better understanding of molecular pathways at mechanistic levels [3]. Beyond enabling the study of diminished expression of a particular gene leading to advancements in understanding molecular pathways, RNAi has demonstrated a potential for being used in therapeutics for treating human diseases $[4,5]$.

Daphnia are freshwater microcrustaceans that inhabit inland waters around the world and often are the critical 
herbivore in aquatic food webs [6, 7]. They have been a major model system in ecology, population genetics, and ecotoxicology for decades due to the ease with which field- and laboratory-based experiments can be conducted. Furthermore, they are cyclic pathenogens, a life cycle that permits genetically diverse natural populations and allows replication of genetically identical individuals through clonal reproduction in the lab [8]. With a fully sequenced genome (D. pulex, [9]), Daphnia has the potential to be a key model organism in molecular ecology and evolution, and is rapidly emerging as a model organism in non-ecological fields including biology of aging [10-15], and neurobiology [16-20]. Methodology for successful gene knockout using TALEN and CRISPER technologies exists for Daphnia [21, 22]. The U.S. National Institutes of Health list Daphnia as a model organism for biomedical research (http://www.nih.gov/ science/models/) citing their extreme phenotypic responses to environmental changes, clonal reproduction, and ecological diversity as advantages in comparison to established biomedical models.

In order to fully realize Daphnia for molecular studies, techniques of experimental genetic manipulation are essential. Currently there is only a single technique described for RNAi in Daphnia [23, 24], which involves microinjecting small dsRNA molecules into the embryos of $D$. pulex and D. magna. Although this system has allowed for the study of some genes regulating embryonic development, there are several drawbacks and limitations to the microinjection method. The microinjection process is technically challenging, involves specialized equipment, thereby making the protocol expressive, tedious and unlikely to be broadly adopted by researchers interested in Daphnia [3, 25]. Currently, microinjection has been performed successfully only in Daphnia embryos. Therefore, this method limits the number of genes one can target as it is mainly applicable to genes expressed during embryonic development. Thus, there is no reliable RNAi method for studying genes expressed later in Daphnia life span, or for achieving knockdown for a specific duration during the life span.

In C. elegans, the problems associated with microinjection for RNAi were mitigated with the introduction of a new technique that involved feeding the worms with bacteria expressing specific dsRNAs [26-28]. This method for systemic RNAi via feeding has been adapted for multiple organisms including, but not limited to, the house cricket (Acheta domesticus), the lepidopteran pest Spodoptera exigua, the brown apple moth (Epiphyas postwittana), the termite Reticulitermes flavipes, and planarians [29-33]. Since the initial report of the systemic RNAi via feeding method in C. elegans, several different genes have been identified as being essential for systemic RNAi via feeding. One of these essential genes is
Sid-1 (systemic RNAi defective), which encodes a transmembrane protein that forms a dsRNA gated channel [34-36]. We searched the recently published Daphnia genome [9] and found that it contains a Daphnid homologue of Sid-1. We also analyzed the D. pulex genome for the various proteins known to be involved in RNAi [1] using the online tool PANTHER (Protein Analysis Through Evolutionary Relationships) that allows for identification of various protein homologs based on domain structures and evolution of protein function in various organisms [37]. We determined that the $D$. pulex genome contains three homologs of Dicer, two homologs of Argonaute, and two homologs of TRBP [9]. Thus, we reasoned that if systemic RNAi via feeding could be achieved for Daphnia, transient gene knockdown experiments would rapidly advance development of this organism as a model system. In our present study, we present a method for efficient RNAi mediated gene knockdown in Daphnia via feeding.

Our study used three different species, D. melanica, $D$. pulex, and D. pulicaria. Although these taxa have different names, they are part of the same species-complex [38-44], hybridization among them is frequent in the wild [45-47], and experimental crosses do not exhibit reproductive isolation [48]. Therefore, they have limited divergence of their genomes, and can be used in complementary assays of genetic function. They were labeled as distinct species by molecular taxonomists based on mitochondrial divergence $[38,39]$, though they also have separate ecological niches, with $D$. melanica specialized for habitats with high UV radiation, and $D$. pulex and $D$. pulicaria specialized to small ponds and large lakes with low UV respectively $[6,42,44,49]$. D. melanica produces melanin as a protective pigment for the high amounts of UV-radiation in its natural habitat $[44,50]$. We used the melanin synthesis pathway of $D$. melanica to develop our RNAi technique, as it provides us with an easily measurable visible phenotype (loss of pigmentation) to assess the effectiveness of gene knockdown. We used $D$. pulex as a comparison because it is the closest relative to $D$. melanica that does not exhibit pigmentation [50].

We selected to target phenoloxidase gene, which encodes an enzyme essential for melanin synthesis in crustaceans, as a target for RNAi $[51,52]$ based on the easily identifiable phenotype it would produce if RNAi was successful. Our results provide evidence for establishment of an easy, feeding-based RNAi method in Daphnia. We demonstrate reduced phenoloxidase mRNA levels, diminished melanin levels resulting in a dramatic phenotype, and reduced survival in response to UV radiation in Daphnia that are fed on bacteria that express a dsRNA specific for phenoloxidase. This is the first demonstration that systemic RNAi is possible in Daphnia. 


\section{Methods}

\section{Daphnia cultures}

Daphnia pulex was isolated from waterbodies in southwest Michigan in 2008 and have since been cultured in the lab. Daphnia melanica were isolated from high altitude alpine lakes in the Sierra Nevada region in eastern California. The isolate used was known as "Sierra", and ND5 mitochondrial gene sequencing confirmed the $D$. melanica species identity $[50,53]$. D. pulex species identity was determined by ldh allozyme characterization (designated "slow-slow") as described previously [54, 55]. D. pulex was maintained at a temperature of $20^{\circ}$ $\mathrm{C}$ with a photoperiod of 12:12 light:dark in a Percival growth chamber. $D$. melanica were maintained at a temperature of $15^{\circ} \mathrm{C}$ with a photoperiod of 16:8 light:dark. All Daphnia were maintained at a concentration of 3 to 5 animals per $250 \mathrm{ml}$ beaker in $100 \mathrm{ml}$ of filtered lake water until experimentation. Lake water was obtained from public access Lake Murray in central South Carolina and was filtered $(1 \mu \mathrm{m})$ before use. Young newborn Daphnia were transferred to a new beaker with fresh water on alternate days. D. pulex cultures were fed every day with vitaminsupplemented algae Ankistrodesmus falcatus at a concentration of 20,000 cells $/ \mathrm{ml}$. D. melanica cultures were fed 20,000 cells/ml of Ankistrodesmus falcatus on alternate days.

\section{Vectors and feeding system}

We selected the L4440 plasmid vector for generating dsRNAs in an inducible manner in E. coli. L4440 plasmid vector (Fig. 1b) was designed by Fire et al. [56]. This vector allows cloning of PCR products between two T7 promoters in opposite orientations. The following two bacterial strains harboring $\lambda \mathrm{DE} 3$ lysogen (a source of $\mathrm{T} 7$ RNA polymerase) were used for generating dsRNA from the recombinant plasmids:

(1) BL21(DE3): ompT hsdS ${ }_{B}\left(r_{B^{-}}-m_{B^{-}}\right)$gal dcm (Novagen) - a strain deficient in lon and ompT proteases and used for efficient production of recombinant proteins.

(2) HT115 (DE3) (W3110, rnc14::DTn10 (Addgene, [57]) - a strain deficient in RNase III and used for efficient production of dsRNAs.

E. coli cells that are (DE3) contain an integrated T7 RNA polymerase ORF under the control of a LacUV5 promoter the cultures can be induced with $2 \mathrm{mM}$ IPTG to produce T7 RNA polymerase. This leads to the production of dsRNA from the PCR product cloned between the two T7 promoters. We also used the plasmid construct L4417, which contains the $5^{\prime}$ half (750 bp) of the GFP ORF cloned between the two T7 promoters (to be used as a negative control in the experiments as it

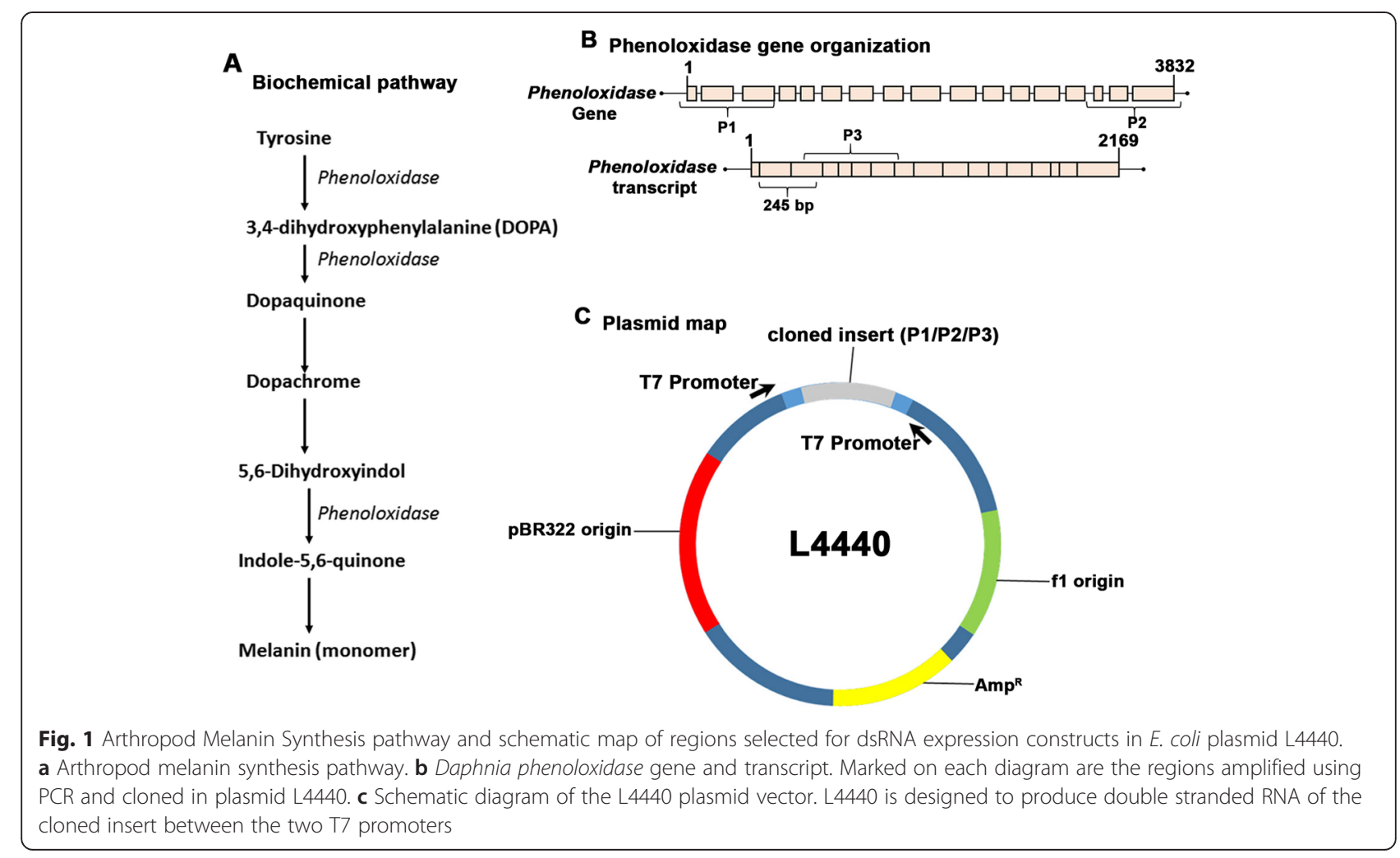


does not have a natural target RNA in Daphnia). All the recombinant plasmids that we generated would produce a dsRNA product of about $800 \mathrm{bp}$ in the bacterial host that expresses T7 RNA polymerase. Both L4440 and L4417 were obtained from Addgene (L4440 Plasmid \#: 1654, L4417 Plasmid \#1649- both gifts from Dr. A. Fire to Addgene).

\section{Target genes and plasmid constructs}

In our current study, we targeted phenoloxidase transcripts for degradation by RNAi. The PCR primers were designed based on D. pulex phenoloxidase (NCBI_GNO_8300047) sequence. We generated several constructs using L4440 plasmid vector to generate dsRNA corresponding to different regions of our target transcripts (Fig. 1c and d). Note for each construct the PCR product was cloned into pGEMT Easy (Promega) and the sequence was confirmed. The following primers were used to generate the PCR products using either the genomic DNA or cDNA as a template. Appropriate restriction enzyme sites were engineered into the $5^{\prime}$ ends of both PCR primers for sub-cloning from pGEMT Easy to L4440. Primers PO245 and GAPDH were used only in qPCR tests of gene expression levels.

P1: (gDNA template) Forward: CACCATGTCAGATT TGCAGC

\section{Reverse: CGCAACATTTGCCTCTTACC}

P2: (gDNA template) Forward: AATTCTTGCCGAT CAAGGTG

Reverse: GCGAAATACGAACGAGGAAA

P3: (cDNA template) Forward: GCGTGGCAGGTTAT

TTTCAT

Reverse: CTTTAGAACGAGCCCAGACG

PO245 (qPCR): Forward: CCATTCAGTCCTAAACC GGA

Reverse: ACCGTCGGAGCATTCTTAAA

GFP: Forward: GCCCGAAGGTTATGTACAGG

Reverse: AAAGGGCAGATTGTGTGGAC

GAPDH: Forward: TTATCACCTCCTCAACTTC

Reverse: CTTCTTCCTTCACTTCTCC

The following abbreviations denote which targeting vector was in the bacteria administered as feed to Daphnia: EV: Empty Vector L4440, GFP: GFP Control (L4417), P1: 5' half of phenoloxidase gene amplified from genomic DNA, P2: 3' half of phenoloxidase gene amplified from genomic DNA, P3: internal region of phenoloxidase mRNA amplified from cDNA, NM: Nonmelanic D. pulex (Clone: RW20), served as a control.

\section{RNAi feeding protocol}

Ten Daphnia aged 4-5 weeks selected for experimentation were placed in $100 \mathrm{ml}$ of filtered lakewater in a $250 \mathrm{ml}$ beaker. E. coli strain BL21(DE3) bacteria transformed with the plasmid of choice (L4440 with one of the inserts indicated in Fig. 1) were grown overnight in Luria Broth (LB) with $2 \mathrm{mM}$ IPTG to induce the expression of T7 RNA polymerase and the dsRNA corresponding to the cloned $\mathrm{PCR}$ product. The $\mathrm{OD}_{600}$ of the overnight cultures was measured, and bacteria from 2.8 $\mathrm{OD}_{600}$ units of overnight culture were pelleted (usually about $1 \mathrm{ml}$ ). The pelleted bacteria were resuspended in $1 \mathrm{ml}$ of filtered lake water and dispensed directly into the beakers, which contained Daphnia, thereby diluting $1 \mathrm{ml}$ of resuspended bacteria in $100 \mathrm{ml}$ of filtered lake water. This corresponds to a final $\mathrm{OD}_{600}$ of 0.028 or about $2.4 \times 10^{7} E$. coli cells in $100 \mathrm{ml}$. This same procedure was repeated for 10 days, with the Daphnia also being fed algae, Ankistrodesmus falcatus, at a concentration of 20,000 cells $/ \mathrm{ml}$ each day for $D$. pulex and and on alternate days for $D$. melanica. The water being changed every other day for $D$. melanica or every day for $D$. pulex. New bacterial culture in fresh LB was prepared for feeding on each feeding day, and fresh algae $(20,000$ cells $/ \mathrm{ml}$ ) added after addition of bacteria to water, thus Daphnia were always fed with a mixture of algae and bacteria. During RNAi feeding regimen, the same photoperiods as stated under Daphnia cultures section were maintained for each species.

Initially we set up experiments to determine the optimal amount of bacteria and feeding duration for achieving effective RNAi. For this purpose, $10 \mathrm{D}$. melanica in $100 \mathrm{ml}$ of lake water were fed on varying amounts of bacteria expressing the phenoloxidase dsRNA (P1) for 12 days. We recorded lethality as well as phenotypic response each day (Table 1) and the bacterial concentration that showed the best phenotype change and the least lethality was selected for analysis of phenoloxidase mRNA knockdown by Real Time PCR.

\section{UV treatment of $D$. melanica}

For the experiments involving the knockdown of phenoloxidase in D. melanica, on the tenth and eleventh days of the bacterial feeding regimen, Daphnia were exposed to UV radiation by using a transilluminator with $312 \mathrm{~nm}$ UVB emission. Daphnia in $250 \mathrm{ml}$ beakers (in $100 \mathrm{ml}$ of water) were placed on the transilluminator. Beakers were arranged such that individual Daphnia were an average $10 \mathrm{~cm}$ from the UV source. Daphnia were exposed to UV for $5 \mathrm{~min}$, and then returned to the Percival chamber. On the day 12 after beginning of bacterial feeding, the Daphnia were sacrificed and assayed for visual phenotypes and harvested to assay melanin content or isolate total RNA. This UV treatment allowed for a rigorous assessment of the RNAi targeting phenoloxidase via feeding method. D. melanica, if stressed, can stop production of melanin synthesis and this may result in false positive scoring of the loss of pigmentation phenotype since the presence of bacteria in water may induce 
Table 1 Optimization of the RNAi feeding regimen

\begin{tabular}{|c|c|c|c|c|c|c|c|c|c|c|}
\hline bacteria & Daphnia & Day 0 & Day & Day & Day & Day & Day & Day & \multirow{2}{*}{$\begin{array}{l}\text { \% } \\
\text { dead }\end{array}$} & \multirow{2}{*}{$\begin{array}{l}\% \\
\text { clear }\end{array}$} \\
\hline $\begin{array}{l}\text { (cells/100 } \\
\text { ml) }\end{array}$ & response & (Start) & 2 & 4 & 6 & 8 & 10 & 12 & & \\
\hline \multirow[t]{4}{*}{$9.6 \times 10^{5}$} & Alive & 10 & 10 & 10 & 10 & 10 & 10 & 9 & \multirow[t]{4}{*}{$10 \%$} & \multirow[t]{4}{*}{$10 \%$} \\
\hline & Dead & 0 & 0 & 0 & 0 & 0 & 0 & 1 & & \\
\hline & Melanic & 10 & 10 & 10 & 10 & 10 & 10 & 8 & & \\
\hline & Clear & 0 & 0 & 0 & 0 & 0 & 0 & 1 & & \\
\hline \multirow[t]{4}{*}{$4.8 \times 10^{6}$} & Alive & 10 & 10 & 10 & 10 & 10 & 9 & 9 & \multirow[t]{4}{*}{$10 \%$} & \multirow[t]{4}{*}{$20 \%$} \\
\hline & Dead & 0 & 0 & 0 & 0 & 0 & 1 & 1 & & \\
\hline & Melanic & 10 & 10 & 10 & 10 & 9 & 8 & 8 & & \\
\hline & Clear & 0 & 0 & 0 & 0 & 1 & 2 & 2 & & \\
\hline \multirow[t]{4}{*}{$2.4 \times 10^{7}$} & Alive & 10 & 10 & 10 & 10 & 9 & 9 & 8 & \multirow[t]{4}{*}{$20 \%$} & \multirow[t]{4}{*}{$60 \%$} \\
\hline & Dead & 0 & 0 & 0 & 0 & 1 & 1 & 2 & & \\
\hline & Melanic & 10 & 10 & 10 & 7 & 6 & 5 & 2 & & \\
\hline & Clear & 0 & 0 & 0 & 3 & 3 & 4 & 6 & & \\
\hline \multirow{4}{*}{$1.2 \times 10^{8}$} & Alive & 10 & 10 & 8 & 6 & 5 & 5 & 4 & \multirow[t]{4}{*}{$60 \%$} & \multirow[t]{4}{*}{$20 \%$} \\
\hline & Dead & 0 & 0 & 2 & 4 & 5 & 5 & 6 & & \\
\hline & Melanic & 10 & 10 & 7 & 5 & 4 & 4 & 2 & & \\
\hline & Clear & 0 & 0 & 1 & 1 & 1 & 1 & 2 & & \\
\hline
\end{tabular}

We fed groups of $10 \mathrm{D}$. melanica in $100 \mathrm{ml}$ of lake water on varying amounts of bacteria expressing the phenoloxidase dsRNA (P1) as indicated in the first column for a duration of 12 days. We recorded lethality (which is listed as the total number of Daphnia that had died from start of the experiment and overall \% dead at the end of the experiment) as well as phenotypic response (which is listed as the total number of live Daphnia displaying a clear carapace on a particular day and overall \% with clear carapace at the end of the experiment). The percentages were calculated based on starting number of individuals (10). Only the living Daphnia were scored for carapace color. The green rows and columns indicate the results representing the bacterial concentration that was selected for analysis of phenoloxidase mRNA levels on day 10. This was a concentration that exhibited the best phenotypic response

some level of stress. By exposing Daphnia to UV radiation, the synthesis of melanin can be induced, overriding any probable down regulation due to stress.

\section{Melanin assay}

The assay was performed as per Hebert and Emery [58]. The body length of Daphnia to be analyzed via the Melanin Assay for their melanin content was measured and then Daphnia were placed in $50 \mu \mathrm{l}$ of $5 \mathrm{M} \mathrm{NaOH}$ and incubated at $40{ }^{\circ} \mathrm{C}$ for 4 days. The melanin content of the resulting solution was determined by measuring optical density at $420 \mathrm{~nm}$ with a plate reader. We performed a melanin standard curve using commercially available bovine melanin. By generating a standard curve, we were able to convert our $\mathrm{OD}_{420}$ values into micrograms of melanin per millimeter of Daphnia [53, 58]. We also measured the melanin content of a nonmelanic Daphnia species as a negative control.

\section{UV sensitivity and survival assay}

We tested Daphnia's ability to survive UV exposure following the loss of pigmentation in response to feeding on bacteria expressing the phenoloxidase dsRNA. Daphnia in control and treatment groups were fed bacteria as described above for 10 days. On the eleventh day, Daphnia were subjected to UV radiation (10 min of UV radiation on the transillimuminator). We subjected the Daphnia a second time to this UV dosage on day 12 and on the thirteenth day examined the viability in control and treatment groups.

\section{Reverse transcriptase (RT)-PCR}

Total RNA was isolated using RNAzol B reagent (TelTest) from 6 to 10 Daphnia following a bacterial RNAi feeding regimen for 10 days. Prior to RNA isolation, for the Daphnia fed on bacteria expressing any of the described dsRNAs, the entire gut was removed from each individual to avoid contamination from the bacteria in the gut that contain the dsRNA. Daphnia were collected in a microcentrifuge tube, rinsed once with $1 \mathrm{ml}$ of PBS, and were homogenized in $0.8 \mathrm{ml}$ of RNAzol B. Total RNA was isolated as per the supplied protocol. cDNA was synthesized using random hexamer primers, $1 \mu \mathrm{g}$ total RNA, 10-20 units M-MuLV reverse transcriptase, $500 \mu \mathrm{M}$ dNTPs, 40 units RNase Inhibitor RNasin (Promega) in appropriate reaction buffer. For each PCR reaction, $2 \mu \mathrm{l}(1 / 10$ th of total) cDNA was used with 50 pmoles each of the forward and reverse primers designed to amplify phenoloxidase PCR product using the Promega GoTaq PCR kit. The following conditions were used for PCR: $95^{\circ} \mathrm{C}$ for $5 \mathrm{~min}$ (initial denaturation), denaturation at $95^{\circ} \mathrm{C}$ for $30 \mathrm{~s}$, annealing at $58^{\circ} \mathrm{C}$ for $30 \mathrm{~s}$, extension at $72^{\circ} \mathrm{C}$ for $30 \mathrm{~s}$ for 27 cycles in order to stay within linear range of amplification. The linear range was determined by varying cycle numbers and performing a densitometric 
analysis of the amplified product. PCR products were separated on a $1 \%$ agarose gel.

\section{Real time PCR}

We first determined the efficiency of the real time PCR reactions with serial dilutions of all cDNAs. Every reaction was performed in triplicate in a total volume of $20 \mu \mathrm{l}$. This included $4 \mu \mathrm{l}$ cDNA, 250nM phenoloxidase or GAPDH primers, and SensiFast Supermix (BioLine). GAPDH was used for normalization. Phenoloxidase and GFP primers were validated by running serial dilutions with a template of known quality and the efficiency of the reactions were determined to be greater than $98 \%$. GAPDH primers were previously validated [53]. In addition, we also ensured that the GAPDH mRNA levels did not change during our treatments prior to performing experimental qRT-PCR for analyzing the phenoloxidase mRNA levels. In all samples analyzed (EV, GFP, P1, P2, P3 and D. pulex), the GAPDH threshold cycle (Ct) value was determined to be $24.5+/-0.10$. This initial standardization was important because GAPDH mRNA levels were reported to change under certain treatment conditions in Daphnia [59]. All reactions were run on a BioRad CFX96 Real Time System C1000 Thermal cycler machine with the following conditions: $95^{\circ} \mathrm{C}$ for $30 \mathrm{~s}, 95^{\circ} \mathrm{C}$ for $5 \mathrm{~s}, 58^{\circ} \mathrm{C}$ for $5 \mathrm{~s}$ for phenoloxidase and $54 \mathrm{C}$ for $5 \mathrm{~s}$ for GFP (the last three steps repeated for 50 cycles), $65^{\circ} \mathrm{C}$ for $5 \mathrm{~s}$, and then $95^{\circ} \mathrm{C}$ for $5 \mathrm{~s}$. We analyzed our data using the Bio-Rad CFX Manager Software with the $2^{-\Delta \Delta C t}$ method. Note that three independent RNA isolations were used from three independent groups of Daphnia to serve as biological replicates.

\section{Statistics}

To determine statistical significance, a two tailed Student's $T$-test assuming equal variance or chi square analysis was performed. Each figure legend denotes $\mathrm{p}$ values as set forth by brackets and special $F$ characters. Note that our alpha level was $p=0.05$.

\section{Results and discussion}

\section{Selection of target}

In order to establish an RNAi method for Daphnia via feeding, we selected target genes that would result in easily identifiable visible phenotypes. A deficiency of phenoloxidase enzyme would result in a reduction of melanin pigment, thus producing a visible loss of pigmentation in D. melanica. As shown in Fig. 1a, we selected to target phenoloxidase gene based on its involvement in several essential steps in the melanin synthesis pathway $[52,60]$. In order to test dsRNAs corresponding to three different regions of phenoloxidase gene for their effectiveness, we used three PCR primer pairs to amplify the indicated regions for sub-cloning into the plasmid vector L4440. As is shown in Fig. 1b, two regions from the phenoloxidase gene (with primers binding in introns of the gene) corresponding to the $5^{\prime}$ region (P1) and 3' region (P2) were selected for PCR amplification. Another primer pair was used with cDNA as a template (therefore no intronic regions would be present), which corresponds to a central region of the phenoloxidase transcript (named P3, Fig. 1b). A different primer pair was used for real time PCR in order to measure changes in phenoloxidase transcript levels (termed PO245, Fig. 1b) after RNAi feeding regimen.

\section{Optimization of the RNAi feeding protocol}

We selected the concentration of bacteria in feed as well as the duration of the RNAi feeding regimen based on our initial experiments to determine the effective dose that would result in the least lethality and a quick phenotypic response (loss of pigmentation in D. melanica). As seen in Table 1, we selected the bacterial dose that resulted in a phenotypic change in 10 days with the least lethality (which was $2.4 \times 10^{7}$ bacterial cells/day/ $100 \mathrm{ml}$ lake water for 10 days). Note that although this concentration and duration was optimal for targeting phenoloxidase in D. melanica, the optimal concentration and duration may have to be determined for specific species and clones of Daphnia as well as for different target genes using the RNAi via feeding system. The method used for this initial optimization would be similar to one we outline here.

\section{GFP dsRNA can be detected in Daphnia after being fed on bacteria expressing GFP dsRNA}

First, we wanted to determine if the dsRNA expressed in bacteria was being effectively delivered to Daphnia after being fed on the bacterial suspension. To analyze this, we fed $D$. melanica bacteria expressing GFP dsRNA. The plasmid L4417 (GFP insert cloned in L4440) produces GFP dsRNA from two complementary RNA strands generated from two T7 promoters. Since GFP is not an endogenous Daphnia gene, the presence of GFP dsRNA in Daphnia would indicate that the dsRNA was delivered from bacteria to Daphnia gut and then to the rest of the body. To test this, RNA isolated from Daphnia fed on L4417 containing bacteria was subjected to reverse transcriptase PCR analysis. Before isolating total RNA for analysis, the guts of all Daphnia were removed carefully to ensure that RNA samples were not contaminated with bacteria from the feed. As is shown in Fig. 2a, GFP dsRNA was detected in Daphnia fed on bacteria with L4417 plasmid (lane 2) but not in Daphnia fed on L4440 (EV, lane 1). L4417 plasmid DNA was used as a template positive control for PCR and generated the expected PCR product (lane 3). In order to ensure that 


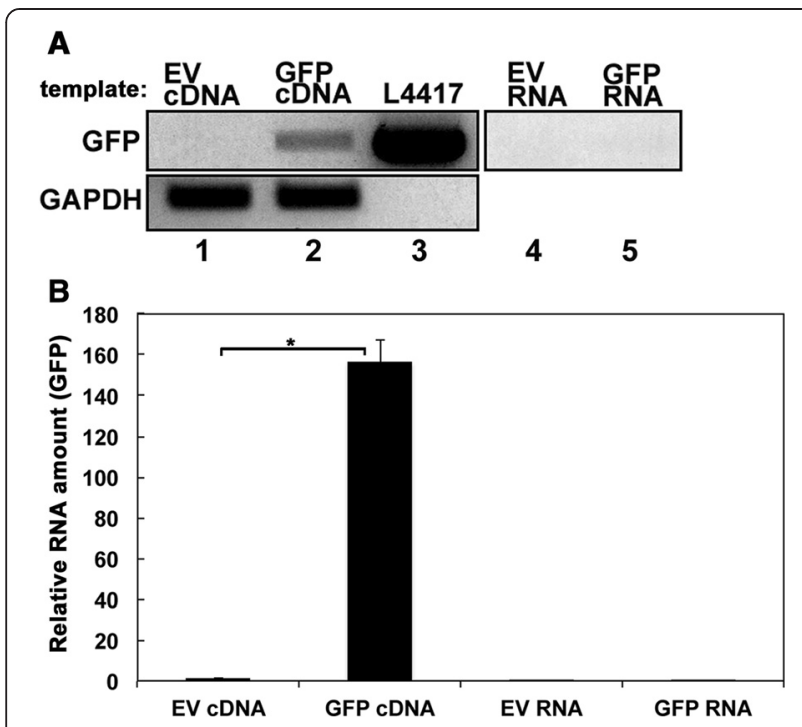

Fig. 2 dsRNA generated in E. coli can be detected in Daphnia. a Reverse transcriptase-PCR data. Lane 1: RNA isolated from Daphnia fed on bacteria containing $L 4440$ without any insert (empty vectorEV), Lane 2: RNA isolated from Daphnia fed on bacteria containing GFP/L4440, Lane 3: GFP/L4440 plasmid DNA (template positive control), Lanes 4 and 5: reverse transcriptase negative control (the negative control without reverse transcriptase ensures lack of plasmid DNA contamination). GAPDH was used as an internal control for ascertaining that equal amounts of Daphnia mRNA were analyzed in lanes 1 and 2. b Quantitative real time PCR data. Real Time PCR was performed with the same samples as in panel $A$. P-value: ${ }^{*}=5 \times 10^{-5}$

there was no plasmid DNA contamination in the RNA preparation originating from bacteria in Daphnia guts, we performed PCR using the isolated RNA (and not cDNA) without the reverse transcriptase step as a template. As seen in lanes 4 and 5, no PCR product was generated in the absence of reverse transcriptase reaction thereby confirming that the PCR product was being generated only from GFP dsRNA (Note: all RNA was treated with DNase before being analyzed). Fig. 2b shows a quantitative reverse transcriptase PCR (qRTPCR) confirming our results from Fig. 2a. The L4417 template positive control was excluded from Fig. $2 \mathrm{~b}$ because as expected it showed an extremely high level of amplification and PCR product. Thus we were able to detect non-endogenous GFP dsRNA in Daphnia fed on bacteria containing the plasmid L4417, demonstrating an effective delivery of dsRNA to Daphnia tissues other than gut. The results conclusively prove that GFP dsRNA is detected in Daphnia tissues other than the gut and indicates effective delivery of dsRNA.

\section{Phenotypic change in $D$. melanica fed on bacteria expressing phenoloxidase dsRNA}

To investigate the effectiveness of RNAi via feeding method, we fed $D$. melanica on bacteria expressing the phenoloxidase dsRNA to target this essential enzyme in melanin biosynthesis pathway. After the RNAi feeding regimen, Daphnia were observed for loss of pigmentation and the percentages of Daphnia exhibiting loss of pigmentation were calculated. Daphnia were grouped either as pigmented or not pigmented. There were some variations in pigmentation loss phenotype and Daphnia showing greater than $50 \%$ loss of pigmentation along the back of the carapace, between the gut and the edge of the carapace, were classified as loss of pigmentation while the ones showing less than $50 \%$ loss were classified as pigmented. We performed this classification by analyzing photos of Daphnia each day of their RNAi feeding regimen. The majority of organisms that showed loss of pigmentation had completely clear carapace with no pigmentation. However, about $20 \%$ of the ones classified as lacking pigmentation had partially clear carapaces with pigmentation apparent in $50 \%$ or less carapace area. Demonstrated in Fig. 3, D. melanica fed on bacteria expressing phenoloxidase dsRNA displayed a remarkable loss of pigmentation (Fig. 3a and b). This phenotype was not seen in $D$. melanica that were fed L4440 plasmid vector containing no inserts (EV) or a plasmid vector expressing GFP dsRNA (L4417). No visible effect of the phenoloxidase dsRNA produced in bacteria was observed in nonmelanic Daphnia (Daphnia pulex, Clone: RW20) when fed on dsRNA producing bacteria. As is shown in Fig. 3a and b, D. melanica with no treatments showed a slight change in phenotype (Black bars, Fig. 3a and b, $6.0+/-5.2 \%$ ), which can be thought as natural variation in pigmentation. D. melanica fed on bacteria with EV (blue bars) and GFP control constructs (green bars) showed a pigmentation loss phenotype in some individuals $(15.6+/-5.9 \%$ and 22 $+/-5.4 \%$ respectively) in comparison to the group with no treatment. $D$. melanica can show reduced melanin synthesis if they become stressed [61]. It is possible that the presence of any dsRNA (a short dsRNA may be produced from the polylinker in EV) could trigger stress signaling and elicit down regulation of melanin production and a minor but noticeable phenotype in a small percentage of individuals. However, D. melanica fed on bacteria expressing phenoloxidase dsRNA displayed a markedly high percentage of individuals with the pigmentation loss phenotype (Red bars, Fig. 3a and b) that was 4-6x greater than the non-target controls. In case of the BL21(DE3) bacterial strain, P1 displayed about $75 \%(n=55)$, P2 displayed $78 \%(n=56)$ and P3 was slightly more variable with about $62 \%(n=47)$ of Daphnia displaying the pigmentation loss phenotype (Fig. 3a). The bacterial strain HT115(DE3) that lacks the RNase III activity exhibited more efficient RNAi (Fig. 3b) as compared to BL21(DE3) strain (Fig. 3a) with P1 displaying about $94 \%(n=16)$, P2 about $86 \%(n=14)$ and P3 

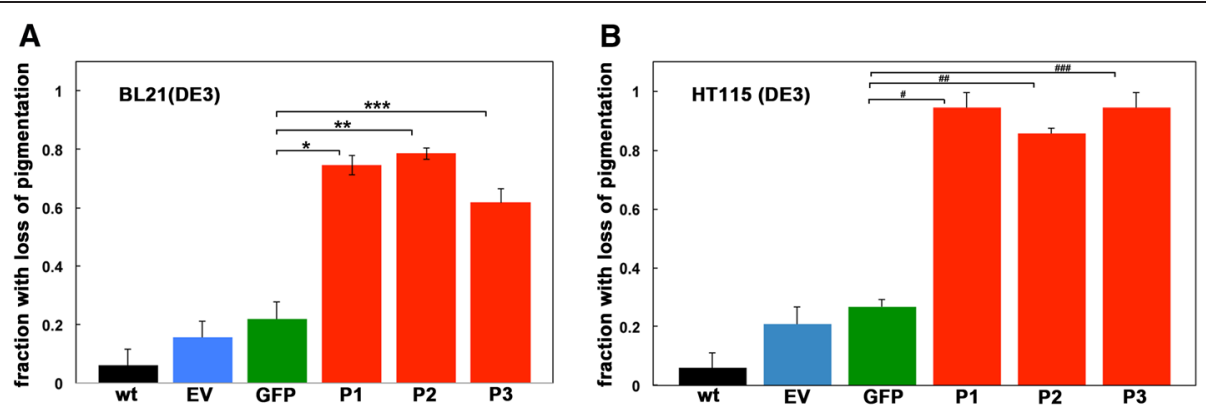

C

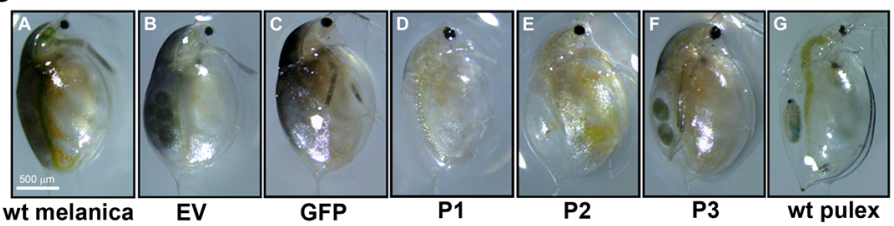

Fig. 3 Daphnia fed on bacteria expressing the phenoloxidase dsRNA demonstrate a loss in melanin pigmentation. a and $\mathbf{b}$ Proportion of Daphnia demonstrating pigmentation loss phenotype after 10 days on an RNAi feeding regimen with BL21(DE3) and HT115(DE3) strain respectively. Following the feeding regimen, Daphnia were UV treated to induce melanin production and then observed under a dissecting scope for pigmentation phenotype. wt (melanica): $n=50$, EV (L4440) $n=51, \mathrm{GFP}(\mathrm{L} 44417): n=50, \mathrm{P} 1$ (P1/L4440): $n=55, \mathrm{P} 2$ (P2/L4440): $n=56, \mathrm{P3}$ (P3/L4440): $n=57$. These abbreviations denote which plasmid was transformed into the bacteria before administering as feed to Daphnia. The data represent five replicate experiments. P-values are as follows: ${ }^{*}=0.0005,{ }^{* *}=0.0032,{ }^{* * *}=0.0002, \#=1.2 \times 10^{-7}, \# \#=2.0 \times 10^{-7}$, and $\# \# \#=$ 0.00016. c Photographic representation of Daphnia from each group as indicated below each panel. The non-melanic species $D$. pulex is shown for comparison

about $94 \%(n=16)$ of Daphnia with loss of pigmentation. Figure $3 \mathrm{c}$ shows a representative individual from each group of Daphnia; either untreated wild type, controls (fed on bacteria harboring EV L4440 or L4417) or fed on bacteria expressing phenoloxidase dsRNA (P1, P2, and P3). The dramatic loss of pigmentation is clearly evident in P1, P2, and P3 groups (panels D, E, and F) as compared to untreated and control samples (panels $\mathrm{A}$, $\mathrm{B}$, and $\mathrm{C}$ ). Panel $\mathrm{G}$ shows a non-melanic $D$. pulex that was fed only on algae $(20,000$ cells/ml) for comparison. These results indicate that RNAi method is successful and it is possible to achieve systemic RNAi via feeding in Daphnia. The bacterial strain HT115(DE3) was more effective in generating the RNAi phenotypes as expected since it is deficient in RNAse III and is known to accumulate dsRNA at high levels compared to BL21(DE3) strain. Although both BL21(DE3) and HT115(DE3) strains work in Daphnia to produce RNAi phenotypes, we recommend using HT115(DE3) strain for future experiments.

During the course of the phenoloxidase RNAi feeding regimen, some progeny organisms born to treated mothers (about 10-15 \%) exhibited clear carapaces, while others in the same clutch were melanic. Due to the unpredictability of the clear offspring being born over the course of the experiment, we did not analyze steady state levels of phenoloxidase mRNA in the progeny with clear carapaces. Once removed from the RNAi feeding regimen, the offspring with clear carapaces always returned to normal melanic pigmentation within the next 4-6 days. It is worth a note that the survival as well as the total offspring number was not affected by the RNAi feeding regimen.

Phenoloxidase transcript levels are diminished in Daphnia fed on bacteria expressing Phenoloxidase dsRNA

Since there was an obvious phenotypic change in $D$. melanica fed on bacteria expressing phenoloxidase dsRNA, we analyzed the phenoloxidase mRNA levels by reverse transcriptase (RT)-PCR (Fig. 4a) as well as quantitative Real Time (qRT)-PCR (Fig. 4b). As is shown in Fig. 4a, phenoloxidase mRNA levels are markedly diminished in Daphnia fed on bacteria expressing phenoloxidase dsRNA (lanes 3-5) as compared to controls (lanes 1-2). Comparing lanes 1 and 2, it appears that there is more phenoloxidase mRNA in the GFP fed Daphnia. The presence of dsRNA may elicit an immune response and phenoloxidase is also a key enzyme in responding to immune insults in Daphnia [52]. Nevertheless, all the phenoloxidase targeting constructs (including those with regions corresponding to genomic DNA) resulted in diminished levels of phenoloxidase mRNA (lanes 3-5). We also used untreated, non-melanic D. pulex (RW20) as a negative control for PCR (lane 6, Fig. 4a). Shown in Fig. 4a and b, D. melanica fed on bacteria expressing phenoloxidase dsRNA displayed levels of phenoloxidase mRNA similar to non-melanic Daphnia (RW20). Thus, when Daphnia are fed on bacteria expressing 


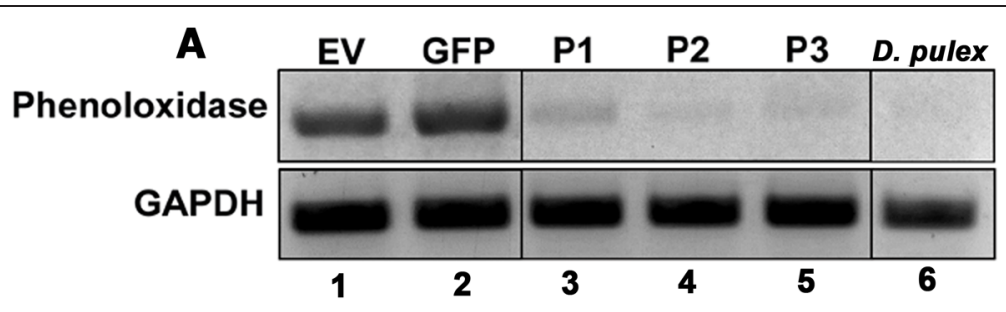

B

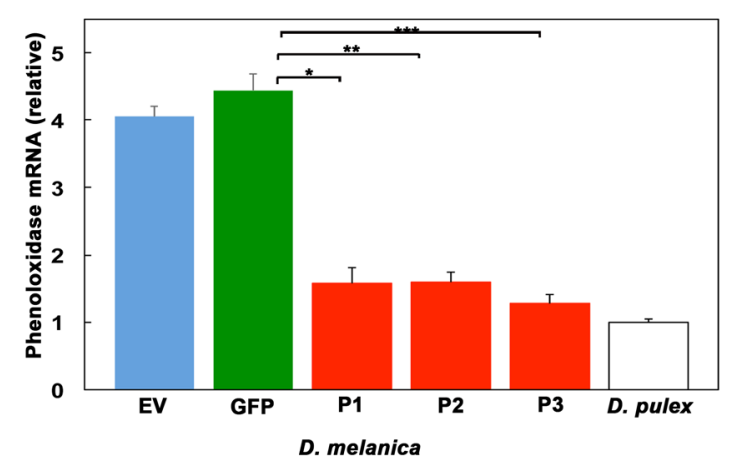

Fig. 4 Phenoloxidase transcript levels are diminished in Daphnia fed on bacteria that express phenoloxidase dsRNA. a Reverse Transcriptase PCR examining the expression of phenoloxidase mRNA. RNA samples are as follows- Lane 1: EV (L4440 without any insert), Lane 2: GFP (GFP/L4440), Lane 3: P1 (P1/L4440), Lanes 4: P2 (P2/L4440), Lane 5: P3 (P3/L4440), and Lane 6: non-melanic D. pulex (isolate RW20) control. GAPDH was used as an internal control for ascertaining that equal amounts of Daphnia mRNA were analyzed in each lane. $\mathbf{b}$ Quantitative real time PCR examining the expression of phenoloxidase mRNA. The abbreviations under each bar denote which plasmid vector was transformed into the bacteria before administering as feed to Daphnia. P-values are as follows: ${ }^{*}=1 \times 10^{-7},{ }^{* *}=7.2 \times 10^{-8}$, and ${ }^{* * *}=9.8 \times 10^{-8}$. Blue bar: EV, Green bar: GFP, red bars: P1, P2, and P3, white bar: non-melanic D. pulex control

phenoloxidase dsRNA, the phenoloxidase mRNA levels decline significantly, thereby demonstrating achievement of very effective RNAi.

\section{Melanin levels are diminished in Daphnia fed on bacteria expressing phenoloxidase dsRNA}

We further validated that RNAi was achieved by measuring melanin content of Daphnia to ensure that the observed phenotypic changes were due to a decline in melanin. The melanin content was determined in Daphnia fed on control bacteria (Fig. 5b, EV and GFP) and Daphnia fed on bacteria expressing the phenoloxidase dsRNA (Fig. 5b, P1, P2, and P3). First we established a standard absorbance curve for various melanin concentrations using commercially available melanin (Fig. 5a). Using the standard curve, the melanin content of Daphnia in experimental groups was determined and is shown in Fig. 5b. Wild type D. melanica (black bar), and the control organisms (blue bar: EV, and green bar: GFP) contained significantly higher amounts of melanin $(32.49+/-1.27 \mu \mathrm{g} / \mathrm{mm}, 29+/-2.5 \mu \mathrm{g} / \mathrm{mm}$, and 35.8 +/ $1.06 \mu \mathrm{g} / \mathrm{mm}$ respectively) than Daphnia fed on bacteria expressing phenoloxidase dsRNA (red bars: P1: 7.2 +/- $1.48 \mu \mathrm{g} / \mathrm{mm}, \mathrm{P} 2: 5.81+/-1.5 \mu \mathrm{g} / \mathrm{mm}$, and P3: 8.21 $+/-2.16 \mu \mathrm{g} / \mathrm{mm}$ of melanin). We included nonmelanic D. pulex as a negative control (white bar: $7.147+/-$ $0.75 \mu \mathrm{g} / \mathrm{mm}$ of melanin). Comparing P1, P2, and P3 to the non-melanic Daphnia, the amount of melanin present is very similar, showing that feeding on bacteria expressing phenoloxidase dsRNA resulted in a marked reduction in melanin levels to bring them to the levels in non-melanic D. pulex (Fig. 5b). Thus, the diminished levels of phenoloxidase mRNA results in a corresponding decrease in melanin content in Daphnia.

\section{Daphnia fed on bacteria expressing phenoloxidase dsRNA} are sensitized to UV radiation

To investigate the functional effect of melanin loss, Daphnia were exposed to UV radiation following the loss of pigmentation after an RNAi feeding regimen. Since melanin protects the DNA damage in response to UV radiation [62], we predicted that the Daphnia exhibiting loss of pigmentation in response to RNAi knockdown of phenoloxidase would be more sensitive to UV. For D. melanica, melanin pigmentation in the wild helps them cope with large amounts of UV radiation they encounter daily $[44,50]$. Daphnia are able to induce the production of melanin following exposure to UV light $[50,53]$. This is particularly important for organisms inhabiting regions high in altitude because their UV exposure tends to be high $[44,50]$. The production of melanin is a protective measure, mitigating the DNA damage resulting from of high doses of UV [50]. Thus, Daphnia were treated with UV radiation and their 

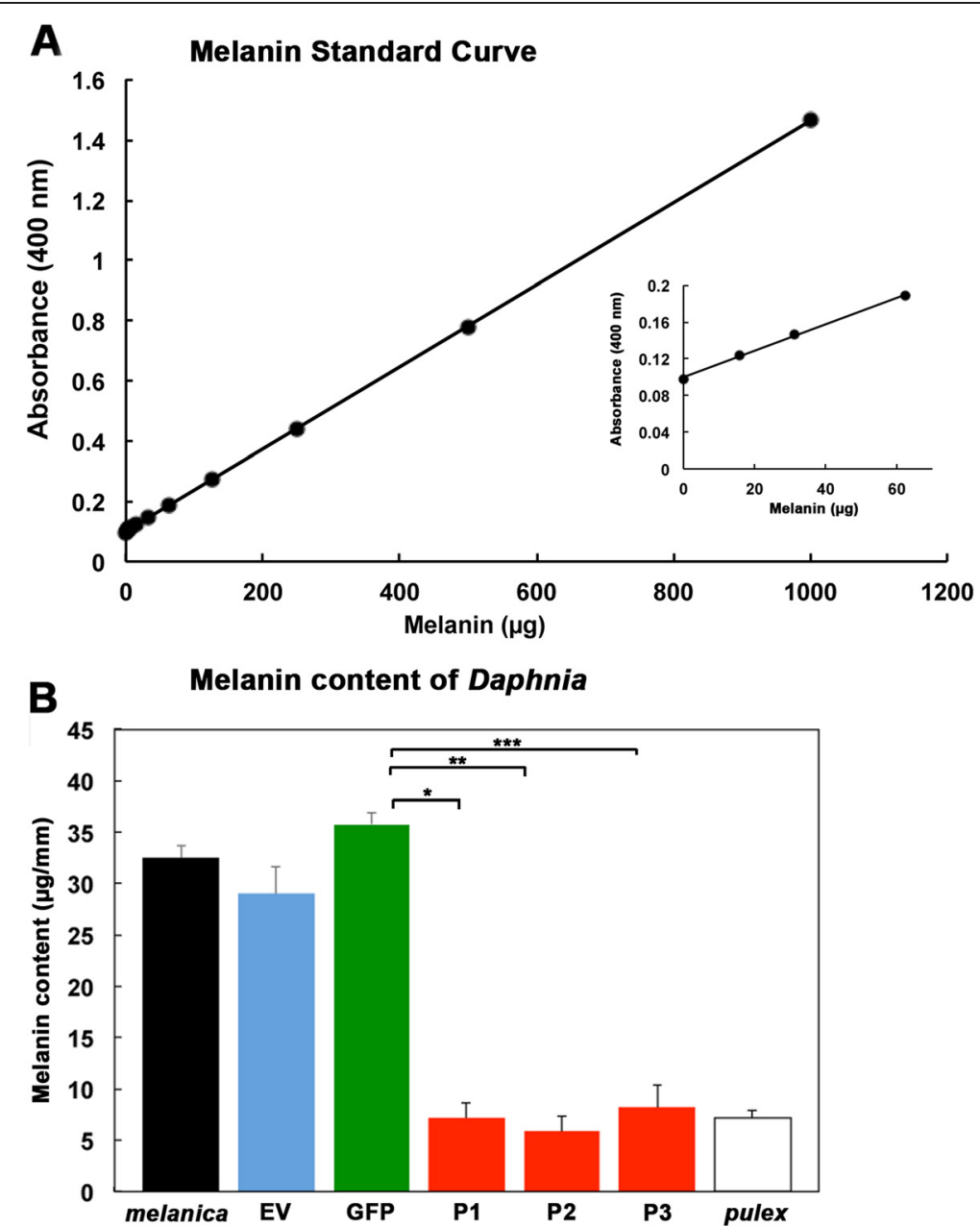

Fig. 5 Melanin content is diminished in D. melanica fed on bacteria expressing phenoloxidase dsRNA. a Melanin standard curve using purified melanin (Sigma). Melanin was dissolved in $5 \mathrm{M} \mathrm{NaOH}$ before making serial dilutions to establish a standard curve. b Melanin Assay performed using extract from various treated Daphnia. wt D. melanica (melanica): $n=14$, EV: $n=15$, GFP (GFP/L4440): $n=14$, P1 (P1/L4440): $n=11$, P2 (P2/ L4440): $n=12$, P3 (P3/L4440): $n=13$, D. pulex RW20 (pulex): $n=20$. Data represents 2 replicate experiments. The abbreviations indicated below the bars denote the plasmid vector that was transformed into the bacteria before administering as feed to Daphnia, except for pulex, which indicates non-melanic control without bacterial feed. P- values are as follows: ${ }^{*}=0.001,{ }^{* *}=0.0002$, and ${ }^{* *}=0.0016$

viability was measured following UV exposure. As shown in Fig. 6, Daphnia that were fed on bacteria expressing phenoloxidase dsRNA displayed a dramatically increased sensitivity to UV with about $50 \%$ lethality (red bars: P1: $45.8+/-2.9 \%$, P2: $57.7+/-3.2 \%$, and P3: $46.7+/-3.5 \%$ death). In contrast to this, wt D. melanica (black bar) and those fed on bacteria with EV (blue bar) or L4417 GFP control (green bar) showed markedly less lethality (EV: $18.1+/-1.4 \%$, GFP: $16.7+/-3.92 \%$ ). The non-melanic $D$. pulex was used as a negative control (white bar) and following UV radiation all $D$. pulex individuals died within $2 \mathrm{~h}(n=43)$. These results further confirm that RNAi targeting of phenoloxidase worked efficiently in Daphnia and exhibited markedly reduced phenoloxidase mRNA, melanin content, and markedly increased UV sensitivity. Thus, the knockdown of phenoloxidase transcript in D. melanica results in a significant reduction in the ability to survive after UV exposure, further validating our RNAi method.

\section{Delivery of dsRNA by feeding is a convenient and reliable RNAi method}

In order to knock down specific gene expression, several methods for the delivery of dsRNA or siRNA have been used in recent years. Among these, RNAi via direct injection of a dsRNA solution is a simple method that works in larger insects due to the easy protocol. It is effective in knocking down expression of target genes in invertebrates such as the cricket G. bimaculatus, the mosquito Aedes aegypti [63], the German cockroach Blattella germanica [64], and the silkworm larvae Bombyx mori [65]. For a small freshwater microcrustacean 


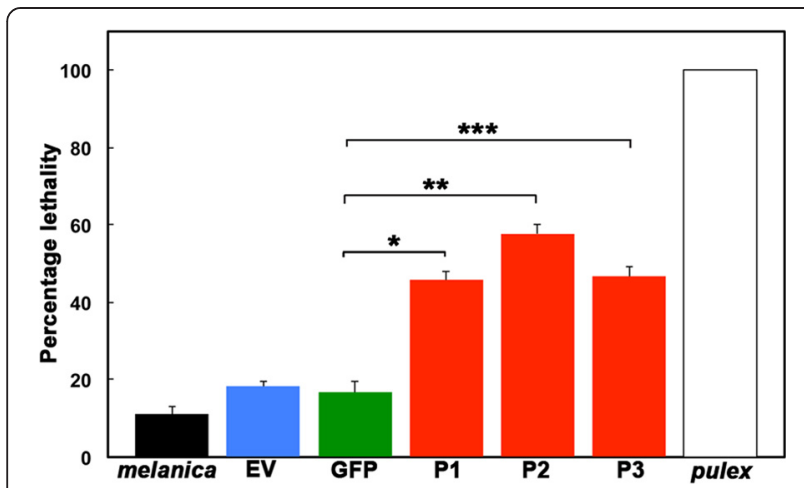

Fig. 6 Enhanced UV sensitivity of D. melanica fed on bacteria expressing phenoloxidase dsRNA. D. melanica were subjected to the RNAi feeding regimen for 10 days and then exposed to UV each day for 2 consecutive days. The percent viability of Daphnia was assessed $24 \mathrm{~h}$ after the second exposure. D. pulex was used as a control and all individuals died within $2 \mathrm{~h}$ of the initial UV insult. wt melanica (melanica): $n=18, \mathrm{EV}: n=22$, GFP (GFP/L4440): $n=24, \mathrm{P} 1$ (P1/L4440): $n=24$, P2 (P2/L4440): $n=26, P 3$ (P3/L4440): $n=30, D$. pulex RW20 (pulex): $n=43$. These abbreviations denote the plasmid that was transformed into the bacteria before administering them as feed to Daphnia. Data represent 3 replicate experiments. $P$-values are as follows: ${ }^{*}=0.0293,{ }^{* *}=0.0028$, and ${ }^{* *}=0.0201$

such as Daphnia, it is difficult to achieve RNAi via injection in adult organisms as injection may result in high mortality since Daphnia will lose viability rapidly if not kept immersed in cool water. Thus, delivering dsRNA by feeding offers several advantages over direct injection of dsRNA method, as it is less labor-intensive, less expensive, and is also applicable for screening a large number of essential Daphnia genes because of its simplicity. Our study also suggests for the first time that RNAi in Daphnia is systemic, and is the first report of phenoloxidase knockdown using RNAi which not only produced significant reduction in mRNA and melanin levels but it also resulted in a marked increase in lethality in response to UV exposure.

It is worth noting that melanin synthesis was restored in D. melanica at about 7 days after bacterial feeding was discontinued, thereby demonstrating that the RNAi was transient and thus this method holds a potential to test the effect of gene knockdown at selected time points during the life span. This may be a very useful feature, especially for aging and longevity research to study the contribution of specific genes in an age-dependent manner. As there was no apparent effect of RNAi bacterial feeding regimen on reproduction in any species we examined, the technique would be widely applicable to most genes. In this regard, it is worth noting that we attempted a knockdown of distal-less and eyeless by the maternal feeding method. A knockdown of distal-less produced no viable progeny, which could be a sign of embryonic lethality specifically due to achieving efficient
RNAi for distal-less. Drosophila distal-less null mutants die as embryos due to defects in development of sensory organs [66]. The knockdown of eyeless was effective and produced a deformed eye phenotype in the progeny as early as in the first clutch after 4-5 days of RNAi feeding regimen. However the organisms exhibiting deformed eyes did not survive for more than a few hours and no RNA could be isolated for analysis of eyeless mRNA levels. Thus, although the preliminary data suggests that targeting mRNAs expressed in developing embryos via feeding the mothers is possible, further experiments are essential to establish the experimental conditions and validation of RNAi effects.

Thus, we present a fast and effective method to achieve gene-specific knockdown in adult organisms as well as developing Daphnia embryos that holds a tremendous potential to become a mainstream method in various types of biological studies that use Daphnia as a model organism.

\section{Conclusions}

We describe a new method to achieve gene specific knockdown by RNAi in Daphnia via feeding. By using $E$. coli cells that express gene-specific dsRNAs as a food additive for adult Daphnia, we can achieve an efficient RNAi for genes that are expressed in adult tissues. This method provides a powerful tool for genetic manipulation of this important model organism for environmental, evolutionary, as well as developmental genomics.

\section{Competing interests}

The authors declare that they have no competing interests.

\section{Authors' contributions}

CAS carried out all the experiments, interpreted the results, performed statistical analysis, and wrote the manuscript. JLD contributed to critical revising of the manuscript. RCP conceived the study, participated in design and coordination of all experiments, and helped to write the manuscript. All authors read and approved the final manuscript.

\section{Acknowledgements}

The authors would like to thank Dr. Michael Pfrender (Department of Biological Sciences, University of Notre Dame, Notre Dame, IN, USA) for kindly providing us D. melanica. This work was supported by National Institutes of Health grant 1R01AG037969-01 awarded to RCP and JLD. The funding agency had no role in study design, data collection and analysis, decision to publish, or preparation of the manuscript.

Received: 22 June 2015 Accepted: 1 October 2015

Published online: 07 October 2015

\section{References}

1. Ipsaro JJ, Joshua-Tor L. From guide to target: molecular insights into eukaryotic RNA-interference machinery. Nat Struct Mol Biol. 2015;22(1):20-8. 2. Ketting RF. The many faces of RNAi. Dev Cell. 2011;20(2):148-61.

3. Perrimon N, Ni JQ, Perkins L. In vivo RNAi: today and tomorrow. Cold Spring Harb Perspect Biol. 2010;2(8):a003640.

4. Deng Y, Wang CC, Choy KW, Du Q, Chen J, Wang Q, et al. Therapeutic potentials of gene silencing by RNA interference: principles, challenges, and new strategies. Gene. 2014;538(2):217-27.

5. Rettig GR, Behlke MA. Progress toward in vivo use of siRNAs-II. Mol Ther. 2012;20(3):483-512. 
6. Benzie J. The genus Daphnia (including daphniopsis) (anomopoda: daphniidae). Guides to the identification of the microinvertebrates of the continental waters of the world. 2005th ed. Leiden: Ghent and Backhuys Publishers; 2005.

7. Peters RH, Bernardi R. Daphnia. Verbania: Istituto Italiano di Idrobiologia; 1987.

8. Zaffagnini F. Reproduction in Daphnia. Mem Ist Ital Idrobiol. 1987;45:245-84.

9. Colbourne JK, Pfrender ME, Gilbert D, Thomas WK, Tucker A, Oakley TH, et al. The ecoresponsive genome of Daphnia pulex. Science. 2011;331(6017):555-61.

10. Dudycha $J$, Hassel C. Aging in sexual and obligately asexual clones of from temporary ponds. J Plankton Res. 2013;35(2):253-9.

11. Lohr JN, David P, Haag CR. Reduced lifespan and increased ageing driven by genetic drift in small populations. Evolution. 2014;68:2494-508.

12. Murthy M, Ram J. Invertebrates as model organisms for research on aging biology. Invertebr Reprod Dev. 2014;59:1-4.

13. Pietrzak B, Bednarska A, Grzesiuk M. Longevity of Daphnia magna males and females. Hydrobiologia. 2008;643:71-5.

14. Schumpert C, Handy I, Dudycha JL, Patel RC. Relationship between heat shock protein 70 expression and life span in Daphnia. Mech Ageing Dev. 2014:139:1-10.

15. Schumpert C, Nelson J, Kim E, Dudycha JL, Patel RC. Telomerase activity and telomere length in Daphnia. PLoS One. 2015;10(5):e0127196.

16. Christie AE, McCoole MD. From genes to behavior: investigations of neurochemical signaling come of age for the model crustacean Daphnia pulex. J Exp Biol. 2012;215(Pt 15):2535-44.

17. McCoole MD, Atkinson NJ, Graham DI, Grasser EB, Joselow AL, McCall NM, et al. Genomic analyses of aminergic signaling systems (dopamine, octopamine and serotonin) in Daphnia pulex. Comparative biochemistry and physiology Part D. Genomics \& proteomics. 2012;7(1):35-58.

18. Toyota K, Miyakawa H, Yamaguchi K, Shigenobu S, Ogino Y, Tatarazako N, et al. NMDA receptor activation upstream of methyl farnesoate signaling for short day-induced male offspring production in the water flea, Daphnia pulex. BMC Genomics. 2015;16:186.

19. Ungerer P, Eriksson BJ, Stollewerk A. Neurogenesis in the water flea Daphnia magna (Crustacea, Branchiopoda) suggests different mechanisms of neuroblast formation in insects and crustaceans. Dev Biol. 2011;357(1):42-52.

20. Weiss LC, Tollrian R, Herbert Z, Laforsch C. Morphology of the Daphnia nervous system: a comparative study on Daphnia pulex, Daphnia lumholtzi, and Daphnia longicephala. J Morphol. 2012;273(12):1392-405.

21. Hiruta C, Ogino Y, Sakuma T, Toyota K, Miyagawa S, Yamamoto T, et al. Targeted gene disruption by use of transcription activator-like effector nuclease (TALEN) in the water flea Daphnia pulex. BMC Biotechnol. 2014;14(1):95.

22. Nakanishi T, Kato Y, Matsuura T, Watanabe H. CRISPR/Cas-mediated targeted mutagenesis in Daphnia magna. PLoS One. 2014;9(5):e98363.

23. Hiruta C, Toyota K, Miyakawa H, Ogino Y, Miyagawa S, Tatarazako N, et al. Development of a microinjection system for RNA interference in the water flea Daphnia pulex. BMC Biotechnol. 2013;13:96.

24. Kato $Y$, Shiga $Y$, Kobayashi K, Tokishita S, Yamagata H, Iguchi T, et al. Development of an RNA interference method in the cladoceran crustacean Daphnia magna. Dev Genes Evol. 2011;220(11-12):337-45.

25. Yu N, Christiaens O, Liu J, Niu J, Cappelle K, Caccia S, et al. Delivery of dsRNA for RNAi in insects: an overview and future directions. Insect Sci. 2013;20(1):4-14

26. Timmons $L$, Court DL, Fire A. Ingestion of bacterially expressed dsRNAs can produce specific and potent genetic interference in Caenorhabditis elegans. Gene. 2001;263(1-2):103-12

27. Timmons L, Fire A. Specific interference by ingested dsRNA. Nature. 1998;395(6705):854

28. Wang J, Barr MM. RNA interference in Caenorhabditis elegans. Methods Enzymol. 2005;392:36-55

29. La Fauce K, Owens L. Suppression of Penaeus merguiensis densovirus following oral delivery of live bacteria expressing dsRNA in the house cricket (Acheta domesticus) model. J Invertebr Pathol. 2013;112(2):162-5.

30. Newmark PA, Reddien PW, Cebria F, Sanchez AA. Ingestion of bacterially expressed double-stranded RNA inhibits gene expression in planarians. Proc Natl Acad Sci U S A. 2003;100 Suppl 1:11861-5.

31. Tian H, Peng H, Yao Q, Chen H, Xie Q, Tang B, et al. Developmental control of a lepidopteran pest Spodoptera exigua by ingestion of bacteria expressing dsRNA of a non-midgut gene. PLoS One. 2009;4(7):e6225.
32. Turner CT, Davy MW, MacDiarmid RM, Plummer KM, Birch NP, Newcomb RD. RNA interference in the light brown apple moth, Epiphyas postvittana (Walker) induced by double-stranded RNA feeding. Insect Mol Biol. 2006:15(3):383-91.

33. Zhou X, Wheeler MM, Oi FM, Scharf ME. RNA interference in the termite Reticulitermes flavipes through ingestion of double-stranded RNA. Insect Biochem Mol Biol. 2008;38(8):805-15.

34. Winston WM, Molodowitch C, Hunter CP. Systemic RNAi in C. elegans requires the putative transmembrane protein SID-1. Science. 2002:295(5564):2456-9.

35. Feinberg $E H$, Hunter CP. Transport of dsRNA into cells by the transmembrane protein SID-1. Science. 2003;301(5639):1545-7.

36. Shih JD, Hunter CP. SID-1 is a dsRNA-selective dsRNA-gated channel. RNA. 2011;17(6):1057-65.

37. Mi H, Muruganujan A, Thomas PD. PANTHER in 2013: modeling the evolution of gene function, and other gene attributes, in the context of phylogenetic trees. Nucleic Acids Res. 2013;41(Database issue):D377-86.

38. Colbourne JK, Crease TJ, Weider LJ, Hebert PDN, Dufresne F, Hobaek A Phylogenetics and evolution of a circumarctic species complex (Cladocera: Daphnia pulex). Biol J Linn Soc Lond. 1998;65:347-65

39. Colbourne JK, Hebert PD. The systematics of North American Daphnia (Crustacea: Anomopoda): a molecular phylogenetic approach. Philos Trans R Soc Lond B Biol Sci. 1996:351(1337):349-60.

40. Cristescu ME, Constantin A, Bock DG, Caceres CE, Crease TJ. Speciation with gene flow and the genetics of habitat transitions. Mol Ecol. 2012;21(6):1411-22.

41. Hebert PDN, Finston TL. A taxonomic reevaluation of North American Daphnia (Crustacea: Cladocera). 2. New species in the Daphnia pulex group from the south-central United States and Mexico. Can J Zool. 1996;74:632-53.

42. Pfrender ME, Spitze K, Lehman N. Multi-locus genetic evidence for rapid ecologically based speciation in Daphnia. Mol Ecol. 2000;9(11):1717-35.

43. Vergilino R, Markova S, Ventura M, Manca M, Dufresne F. Reticulate evolution of the Daphnia pulex complex as revealed by nuclear markers. Mol Ecol. 2011;20:1191-207.

44. Mlner BE, Knapp R, Colbourne JK, Pfrender ME. Evolutionary history of alpine and subalpine Daphnia in western North America. Freshw Biol. 2013:58:1512-22.

45. Hebert PDN, Finston TL. Macrogeographic patterns of breeding system diversity in the Daphnia pulex group from the United States and Mexico. Heredity (Edinb). 2001;87:153-61.

46. Hebert PDN, Schwartz SS, Ward RD, Finston TL. Macrogeographic patterns of breeding system diversity in the Daphnia pulex group. 1. Breeding systems of Canadian populations. Heredity (Edinb). 1993;70:148-61.

47. Hebert PDN, Ward RD, Weider LJ. Clonal diversity patterns and breedingsystem variation in Daphnia pulex, an asexual-sexual complex. Evolution. 1988:42:147-59

48. Heier CR, Dudycha JL. Ecological speciation in cyclic parthenogens: Sexual capability of experimental hybrids between Daphnia pulex and Daphnia pulicaria. Limnol Oceanogr. 2009;54:492-502.

49. Dudycha JL. Mortality dynamics of Daphnia in contrasting habitats and their role in ecological divergence. Freshw Biol. 2004;49(5):505-14.

50. Miner BE, Kerr B. Adaptation to local ultraviolet radiation conditions among neighbouring Daphnia populations. Proc Biol Sci. 2011;278(1710):1306-13.

51. Adachi K, Wakamatsu K, Ito S, Miyamoto N, Kokubo T, Nishioka T, et al. An oxygen transporter hemocyanin can act on the late pathway of melanin synthesis. Pigment Cell Res. 2005;18(3):214-9.

52. Labbe P, Little TJ. ProPhenolOxidase in Daphnia magna: cDNA sequencing and expression in relation to resistance to pathogens. Dev Comp Immunol. 2009;33(5):674-80

53. Scoville AG, Pfrender ME. Phenotypic plasticity facilitates recurrent rapid adaptation to introduced predators. Proc Natl Acad Sci U S A 2010;107(9):4260-3.

54. Abastado JP, Miller PF, Jackson BM, Hinnebusch AG. Suppression of ribosomal reinitiation at upstream open reading frames in amino acidstarved cells forms the basis for GCN4 translational control. Mol Cell Biol. 1991;11(1):486-96.

55. Hebert PDN, Beaton MJ, Schwartz SS, Stanton DJ. Polyphyletic origins of asexuality in Daphnia pulex.1. Breedingsystem variation and levels of clonal diversity. Evolution. 1989;43:1004-15. 
56. Fire A, Xu S, Montgomery MK, Kostas SA, Driver SE, Mello CC. Potent and specific genetic interference by double-stranded RNA in Caenorhabditis elegans. Nature. 1998;391(6669):806-11.

57. Dasgupta S, Fernandez L, Kameyama L, Inada T, Nakamura Y, Pappas A, et al. Genetic uncoupling of the dsRNA-binding and RNA cleavage activities of the Escherichia coli endoribonuclease RNase III-the effect of dsRNA binding on gene expression. Mol Microbiol. 1998;28(3):629-40.

58. Hebert PDN, Emery CJ. The adaptive significance of cuticular pigmentation in Daphnia. Funct Ecol. 1990;4:703-10.

59. Spanier KI, Leese F, Mayer C, Colbourne JK, Gilbert D, Pfrender ME, et al. Predator-induced defences in Daphnia pulex: selection and evaluation of internal reference genes for gene expression studies with real-time PCR. BMC Mol Biol. 2010;11:50.

60. Battistella S, Bonivento P, Amirante G. Hemocytes and immunological reactions in crustaceans. Ital J Zool. 2009;63(4):337-43.

61. Tollrian R, Heibl C. Phenotypic plasticity in pigmentation in Daphnia induced by UV radiation and fish kairomones. Funct Ecol. 2004;18(4):497-502.

62. Brenner M, Hearing VJ. The protective role of melanin against UV damage in human skin. Photochem Photobiol. 2008;84(3):539-49.

63. Drake LL, Price DP, Aguirre SE, Hansen IA. RNAi-mediated gene knockdown and in vivo diuresis assay in adult female Aedes aegypti mosquitoes. J Vis Exp. 2012;65:e3479.

64. Martin D, Maestro O, Cruz J, Mane-Padros D, Belles X. RNAi studies reveal a conserved role for RXR in molting in the cockroach Blattella germanica. J Insect Physiol. 2006;52(4):410-6.

65. Liu J, Smagghe G, Swevers L. Transcriptional response of BmToll9-1 and RNAi machinery genes to exogenous dsRNA in the midgut of Bombyx mori. J Insect Physiol. 2013;59(6):646-54.

66. Panganiban G. Distal-less function during Drosophila appendage and sense organ development. Dev Dyn. 2000;218(4):554-62.

\section{Submit your next manuscript to BioMed Central and take full advantage of:}

- Convenient online submission

- Thorough peer review

- No space constraints or color figure charges

- Immediate publication on acceptance

- Inclusion in PubMed, CAS, Scopus and Google Scholar

- Research which is freely available for redistribution 